CASE REPORT

\author{
J. Sanahuja \\ S. Ordoñez-Palau \\ R. Begué \\ L. Brieva \\ D. Boquet
}

\section{Primary Sjögren Syndrome with Tumefactive Central Nervous System Involvement}

SUMMARY: Brain MR imaging abnormalities in primary Sjögren syndrome (pSS) are generally discrete white matter lesions. We describe a 50-year-old woman with recurrent neurologic deficits. MR imaging revealed a large brain lesion. A diagnosis of pSS was made on the basis of clinical features, positive anti-Ro and anti-La antibodies, abnormal Schirmer test findings, and salivary gland scintigraphy. The patient was treated with oral prednisone with good response. Large tumefactive brain lesions are a complication of pSS.
$\mathbf{S}_{\mathrm{p}}^{\mathrm{i}}$ jögren syndrome is an autoimmune disorder that may be primary or secondary to other connective tissue diseases. It is characterized by lymphocytic infiltration and destruction of salivary and lachrymal glands leading to xerostomia and xerophthalmia. Similar mononuclear infiltrates or vasculitic lesions can have extraglandular manifestations. ${ }^{1}$ The prevalence of central nervous system (CNS) disease and MR imaging abnormalities in primary Sjögren syndrome (pSS) is controversial. $^{2-4}$ We present a patient with pSS and a pseudotumoral lesion in the brain.

\section{Case Report}

A 50-year-old woman was referred to our unit with a 6-month history of headache associated with vomiting, impaired visual perception in left visual fields, and difficulty retrieving some words. The patient had a 1-year history of arthralgias, dry mouth, and a 3-kg weight loss. Findings of a neurologic examination were normal. Blood analysis showed antinuclear antibody titers of $1 / 160$ and positive anti-Ro and anti-La antibodies. Findings of serologic tests were negative. MR imaging revealed a demyelinating-inflammatory lesion in the right temporo-occipital white matter. Cerebral angiography did not demonstrate vasculitis. CSF did not show oligoclonal bands. Visual evoked potentials were normal. Symptoms and the radiologic lesion improved spontaneously within 1 month.

Three months later, the patient was admitted because of left facial and brachial paresis and left hemibody hypoesthesia. A new MR imaging showed a large right frontoparietal lesion with ring contrast enhancement surrounding edema and notable mass effect, without restricted diffusion (Fig $1 A,-B$ ). Findings of thoracoabdominal CT, mammography, and tumor markers were negative. Schirmer test results were $<2 \mathrm{~mm}$. Salivary gland scintigraphy was compatible with Sjögren syndrome grade 3 . A diagnosis of pSS was made according to accepted criteria. ${ }^{5}$ Oral prednisone $1 \mathrm{mg} / \mathrm{kg}$ daily was started with dramatic clinical and radiologic improvement (Fig $1 C,-D$ ). In the subsequent months, the oral prednisone dosage was tapered to $5 \mathrm{mg}$ daily.

The patient remained stable until 2 years later, when she experienced right hemifacial paresthesias. MR imaging revealed 2 small hyperintense lesions in the bulb and in the cervical spinal cord on T2-

Received April 4, 2008; accepted after revision May 9

From the Departments of Neurology (J.S., L.B.), Rheumatology (S.O.-P., D.B.), and Radiology-Institut de Diagnòstic per la Imatge (R.B.), Hospital Universitari Arnau de VilanovaIRBLleida, Lleida, Spain.

Please address correspondence to Jordi Sanahuja, MD, Department of Neurology, Hospital Universitari Arnau de Vilanova, Av. Alcalde Rovira Roure 80, 25006 Lleida, Spain; e-mail: jsanahuja@comll.cat

DOI 10.3174/ajnr.A1204 weighted images. Her oral prednisone dosage was increased to 1 $\mathrm{mg} / \mathrm{kg}$ daily again, and hydroxychloroquine was added with good response.

\section{Discussion}

The frequency and type of CNS involvement in pSS is a matter of discussion. The reported prevalence of CNS manifestations ranges from $0 \%$ to $60 \% .^{2-4}$ In a recent cohort study of 400 patients, only $1 \%$ presented with CNS complications. ${ }^{1}$ Peripheral nervous system (PNS) disease is considered the most common neurologic manifestation in pSS. However, the largest study of patients with pSS with neurologic manifestations described CNS manifestations in $68 \%$ of patients, a proportion similar to that of PNS involvement in the same study. ${ }^{6}$

MR imaging abnormalities are common in pSS. These are usually multiple hyperintense areas in the subcortical and periventricular white matter on T2-weighted and fluid-attenuated inversion recovery (FLAIR) sequences. ${ }^{2,3}$ However, MR imaging lesions are generally smaller and less pronounced in pSS than in multiple sclerosis (MS) or systemic lupus erythematosus. ${ }^{2}$ Some authors have reported an increased frequency of pSS in patients with MS. ${ }^{3}$ Older age, PNS or cranial nerve involvement, spinal cord MR imaging lesions spanning multiple spinal segments, MR imaging cortical brain lesions, absence of lesions in the corpus callosum, or lack of oligoclonal bands are atypical in MS and are characteristic of pSS. ${ }^{7}$

Our patient had a tumorlike lesion with a notable mass effect. Tumefactive or pseudotumoral lesions have been described in other autoimmune CNS diseases like MS. ${ }^{8-10}$ Differential diagnosis includes lymphoma, glioma, metastases, abscesses, parasitary cysts, adrenoleukodystrophy, histiocytosis X, progressive multifocal leukoencephalopathy, disseminated encephalomyelitis, and Balò-Marburg disease. ${ }^{8}$

The pathogenesis of CNS damage in pSS remains unknown. Many authors suggest an underlying mechanism of ischemia secondary to small vessel vasculitis. The clinical course, type of MR imaging lesions, and cerebral angiography results in our patient are not consistent with a vasculitic process. Lymphocytic infiltration can produce extraglandular manifestations in pSS, ${ }^{1}$ and it has also been described in the CNS. Bakchine et $\mathrm{al}^{11}$ reported a pathologic case with ataxia, oculomotor paralysis, and seizures and a large lymphocytic infiltrate at postmortem examination. Pseudolymphoma or pseudotumoral lesions affecting several organs like the liver, kidney, lung, pancreas, and choroid plexus have been reported in patients with pSS. ${ }^{12-15}$ In our patient, improvement with corticosteroids, the pattern of contrast enhancement thicker 

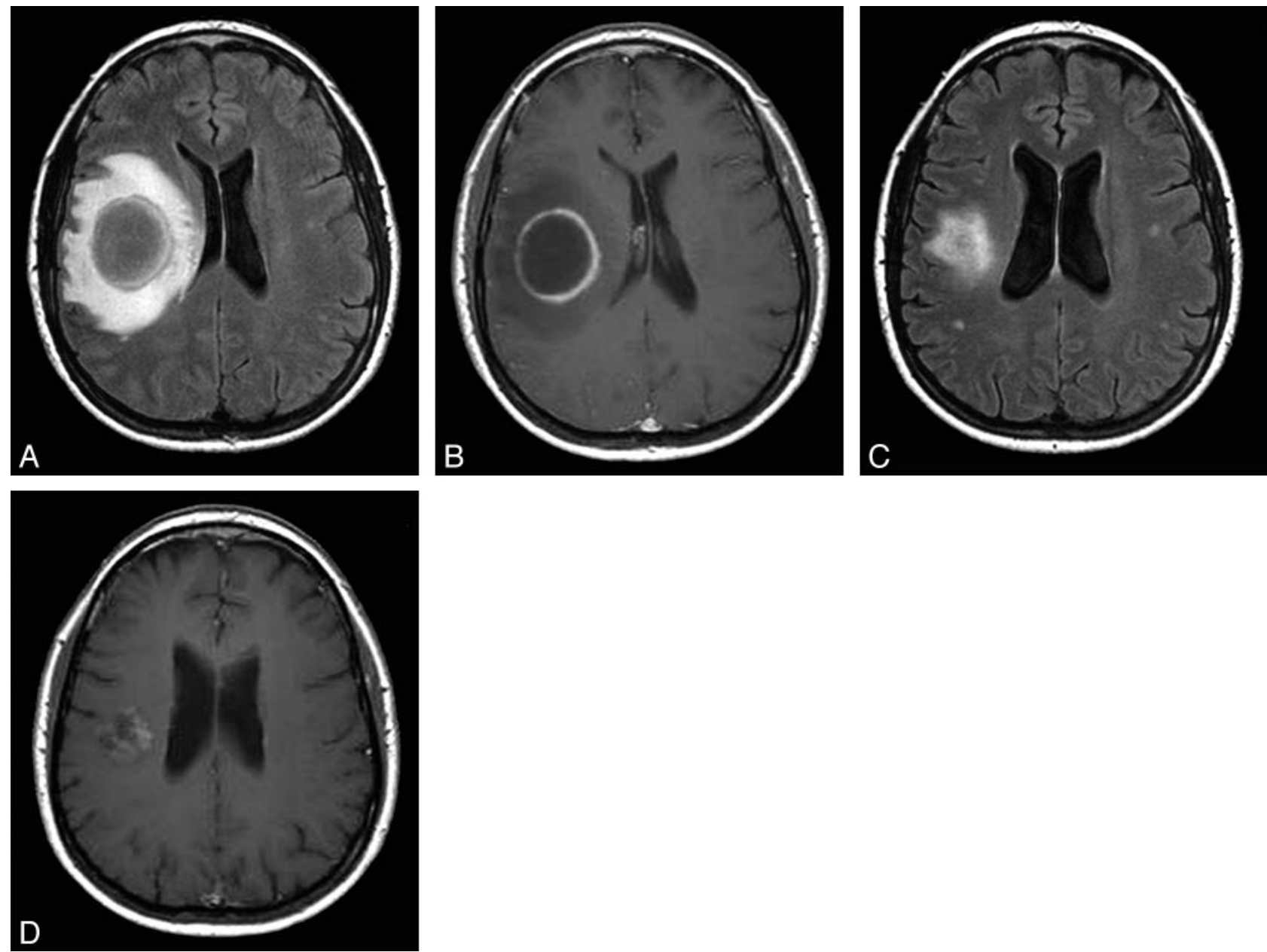

Fig 1. $A$ and $B$, Axial FLAIR $(A)$ and postgadolinium T1-weighted $(B)$ images show a ring-enhancing tumefactive lesion with edema and mass effect. $C$ and $D$, Axial FLAlR $(C)$ and postgadolinium T1-weighted $(D)$ images 1 month after treatment. The pattern of contrast enhancement thicker medially suggests demyelination. The lesion does not show restricted diffusion on diffusion-weighted images and is hyperintense on the apparent diffusion coefficient images.

medially, and a relapsing-remitting course of the lesions suggest an inflammatory-demyelinating mechanism as the underlying pathogenic process.

\section{References}

1. Garcia-Carrasco M, Ramos-Casals M, Rosas J, et al. Primary Sjogren syndrome: clinical and immunologic disease patterns in a cohort of 400 patients. Medicine (Baltimore) 2002;81:270-80

2. Morgen K, McFarland HF, Pillemer SR. Central nervous system disease in primary Sjögren syndrome: the role of magnetic resonance imaging. Semin Arthritis Rheum 2004;34:623-30

3. Soliotis FC, Mavragani CP, Moutsopoulos HM. Central nervous system involvement in Sjögren's syndrome. Ann Rheum Dis 2004;63:616-20

4. de Seze J, Delalande S, Vermersch P. Neurological manifestations in Sjogren syndrome. Rev Med Interne 2005;26:624-36

5. Vitali C, Bombardieri S, Jonsson R, et al, for the European Study Group on Classification Criteria for Sjögren's Syndrome. Classification criteria for Sjögren's syndrome: a revised version of the European criteria proposed by the American-European Consensus Group. Ann Rheum Dis 2002;61:554-58

6. Delalande S, de Seze J, Fauchais AL, et al. Neurologic manifestations in primary
Sjögren syndrome: a study of $\mathbf{8 2}$ patients. Medicine (Baltimore) 2004;83:280-91

7. Rosenbaum R, Barkhuizen A. Primary Sjögren's syndrome. In: Noseworthy $\mathrm{JH}$, ed. Neurological Therapeutics: Principles and Practice. 2nd ed. London, UK Informa Healthcare; 2006:1587-91

8. Comi G. Multiple sclerosis: pseudotumoral forms. Neurol Sci 2004;25:S374-79

9. Khoshyomn S, Braff SP, Penar PL. Tumefactive multiple sclerosis plaque. I Neurol Neurosurg Psychiatry 2002;73:85

10. Selkirk SM, Shi J. Relapsing-remitting tumefactive multiple sclerosis. Mult Scler 2005;11:731-34

11. Bakchine S, Duyckaerts C, Hassine L, et al. Lesions neurologiques centrals et peripheriques au cours d'un syndrome de Gougerot-Sjogren primitif. Rev Neurol 1991;147:368-75

12. Okubo H, Maekawa H, Ogawa K, et al. Pseudolymphoma of the liver associated with Sjögren's syndrome. Scand J Rheumatol 2001;30:117-19

13. Cacoub P, Ginsburg C, Tazi Z, et al. Sjögren's syndrome with acute renal failure caused by renal pseudolymphoma. Am J Kidney Dis 1996;28:762-66

14. Eckstein RP, Hollings RM, Martin PA, et al. Pancreatic pseudotumor arising in association with Sjögren's syndrome. Pathology 1995;27:284-88

15. Chang Y, Horoupian DS, Lane B, et al. Inflammatory pseudotumor of the choroid plexus in Sjögren's disease. Neurosurgery 1991;29:287-90 